\title{
TRATAMIENTO COGNITIVO-CONDUCTUAL DE LAS IDEAS DELIRANTES DE UN PACIENTE ESQUIZOFRÉNICO
}

\author{
Carlos Cuevas y Salvador Perona \\ Unidad de Rehabilitación Selud Mental Virgen del Rocio, Sevilla
}

\begin{abstract}
RESUMEN
En este trabajo presentamos un caso en el que se realiza el análisis funcional y tratamiento cognitivo-conductual de las ideas delirantes de un paciente esquizofrénico. Los resultados muestran una disminución significativa de la convicción del paciente en tales ideas delirantes, asi como una mejora de su funcionamiento personal y social. Los resultados del seguimiento muestran que los beneficios se mantienen a los $1,3,6,12$, 24,36 y 48 meses de finalizado el tratamiento.
\end{abstract}

Palabras clave: Esquizofrenia, delirios, terapia de conducta, terapia cognitivoconductual, rehabilitación psiquiátrica.

\begin{abstract}
In this paper we present a case of a functional analysis and a cognitive-behavioral intervention to modify delusions in a schizophrenic patient. The results show a significative decreasing of delusions and an improvement of his social and personal performance. The results of the follow-up show these benefits still hold $1,3,6,12,24,36$ y 48 months after the end of the treatment.
\end{abstract}

Key words: Schizophrenia, delusions, behavior therapy, cognitive-behavioral therapy, psychosocial rehabilitation.

Correspondencia: Cartos Cuevas, Unidad de Rehabilitación de Salud Mental Virgen del Rocio, Avda. Kansas City, 32, bajo, 41007 Sevilla. 


\section{INTRODUCCIÓN}

Según el Manual Diagnóstico y Estadístico de los Trastornos Mentales, DSM-IV (American Psychiatric Association, 1994), una idea delirante es una "falsa creencia basada en una inferencia incorrecta relativa a la realidad externa que es firmemente sostenida, a pesar de lo que casi todo el mundo cree y a pesar de cuanto constituye una prueba o evidencia incontrovertible y obvia de lo contrario. La creencia no está aceptada ordinariamente por otros miembros de la subcultura o cultura a la que pertenece el sujeto" (p.783). Los delirios están presentes en una amplia variedad de trastornos psicológicos, neurológicos y médicos. Dentro de los trastornos mentales son característicos, entre otras condiciones, de las esquizofrenias. Existen diversas explicaciones psicológicas actuales sobre los delirios: la teoría perceptiva de Maher, el modelo preliminar de Garety sobre los procesos de juicio involucrados en la formación del delirio, las teorias atribucionales y las teorias sobre el contenido de los delirios (Baños y Belloch, 1995). Así mismo, posibles disfunciones cerebrales, variables de personalidad, variables de mantenimiento de la autoestima, emociones, experiencias inusuales, sobrecargas cognitivas prolongadas, déficits atencionales, variables interpersonales y variables situacionales, se señalan como elementos que pueden intervenir en la formación de ideas delirantes.

Al abordar el concepto de idea delirante, diversos autores han propuesto una serie de dimensiones que suponen, entre otros, cinco continuos que van desde las creencias normales hasta las patológicas (Baños y Belloch, 1995). Ello supondría sustituir la visión ampliamente aceptada de los delirios como fenómenos discretos por otra que los considere como partes más o menos extremas a lo largo de diferentes dimensiones. Así, modificable/inmodificable, grado de convicción leve/intenso, presencia/ausencia de apoyos culturales, no preocupación/preocupación y plausible/implausible constituirian los extremos de continuos en los que cabe estudiar las creencias y los juicios.

Desde un punto de vista psicológico, los delirios pueden entenderse como una reacción e intento de dar sentido a determinadas experiencias desconcertantes, e incluso amenazantes, que sufren los pacientes esquizofrénicos.
Eliminar, o paliar, posibles sensaciones de desconcierto, y dar un "sentido" a emociones anteriormente indefinidas, dotaria al delirio de funcionalidad. Así mismo, a los delirios se les puede atribuir otra posible función, defensiva, que les haria más comprensibles, al "proteger" al paciente de la intensa perturbación emocional procedente de evaluaciones negativas "sobre si mismo y sobre los demás" (Chadwick, Birchwood y Trower, 1996).

Al igual que cualquier otra manifestación del comportamiento humano, los síntomas esquizofrénicos, independientemente de que sean elementos constitutivos de un síndrome psiquiatri$c o$, son susceptibles de un análisis funcional y de una evaluación conductual a partir de la cual pueden diseñarse intervenciones especificas a las necesidades de cada paciente (Cuevas y Perona, 1995). Así, diferentes técnicas cognitivoconductuales, tradicionalmente aplicadas a otros trastornos psicológicos, se están adaptando y actualmente utilizando en el tratamiento de la esquizofrenia. Si bien, el tratamiento de elección para la sintomatología psicótica positiva son los neurolépticos, las intervenciones conductuales pueden ser empleadas como complemento en aquellos casos en que los síntomas esquizofrénicos no responden a la medicación y se mantienen perturbaciones en el funcionamiento psicosocial del individuo (Perona y Cuevas, 1997); también, pueden constituir una alternativa terapéutica en aquellos casos de hipersensibilidad a los efectos secundarios de los fármacos, 0 de insuficiente adherencia al tratamiento medicamentoso. No obstante, a la hora de aplicar técnicas cognitivo-conductuales a las personas con psicosis es preciso tener en cuenta la naturaleza de los problemas de estos pacientes: grado de severidad importante, heterogeneidad del trastorno, presencia de déficits cognitivos, posible hipersensibilidad a la activación emocional, posibles dificultades que entorpezcan una adecuada relación terapéutica y la peculiar percepción que del mundo pueden mantener estos pacientes (Fowler, 1996).

Tradicionalmente, la terapia cognitiva se ha propuesto modificar las cogniciones de los sujetos, a través de una combinación de técnicas: la disputa verbal y las pruebas de realidad (Beck, Rush, Shaw y Emery, 1979). En línea con la consideración de los delirios como fenómenos continuos, desde hace algunos años se desa- 
rrollan intervenciones cognitivo-conductuales que han mostrado cierto poder y eficacia para reducir esta sintomatologia en sujetos esquizofrénicos (Por ejemplo, Lowe y Chadwick, 1990; Alford y Beck, 1994; Chadwick y Lowe, 1994; Birchwood y Tarrier, 1995; Kingdon y Turkington, 1995; Chadwick et al. 1996).

El tratamiento cognitivo-conductual de los delirios presenta como objetivo, reducir la convicción en las ideas delirantes y con ello la perturbación emocional asociada, así como la discapacidad social; y ello basándose en métodos que pretenden debilitar tanto las inferencias delirantes del sujeto, como sus creencias evaluativas. En líneas generales el proceso su-pone los siguientes pasos:

$\left.1^{\circ}\right)$ Realización de una serie de entrevistas preliminares con la finalidad de establecer un buen rapport con el paciente.

$\left.2^{\circ}\right)$ Obtención de una línea base, para acumular información sobre sus creencias, cogniciones evaluativas e ideas delirantes (y su posible funcionalidad psicologica); evaluando, a través de medidas subjetivas, el grado de convicción que el paciente verbaliza sobre sus ideas, la preocupación o tiempo que pasa pensando en ellas, y asi mismo, valorando las evidencias actuales y pasadas a favor $y$ en contra de tales ideas.

$3^{\circ}$ ) El enfoque cognitivo de la evaluación de los delirios se enmarca dentro del modelo $A B C$ (acontecimientos activadores; creencias delirantes y evaluativas; y consecuencias emocionales y de conducta), y define los problemas (en " $\mathrm{C}^{\text {) }}$ ) como cuestiones de perturbación y trastorno, más que presentar como problema las " $A$ " (voces...) o " $B$ " (delirios). Este enfoque, adaptado al nivel de funcionamiento cognitivo de cada individuo, es transmitido al paciente.

$\left.4^{\circ}\right)$ El tratamiento se compone del desafío o disputa verbal (para los pensamiento delirantes y para los pensamientos evaluativos), y de la comprobación de la realidad (para los pensamiento delirantes). La disputa verbal o desaffo, consiste en: el cuestionamiento de la evidencia aportada para las creencias y delirios; el cuestionamiento de la consistencia interna y la plausibilidad de dichos contenidos; la redefinición del delirio como respuesta comprensible, como forma de dar sentido a unas experiencias especificas y; la construcción de explicaciones alternativas y significativas para el paciente. A través de un proceso de discusión colaborativa y de comprobación directa de la realidad, se pretenderia el rechazo del delirio a favor de un marco explicativo alternativo menos perturbador y más adaptativo.

En este trabajo presentamos un estudio de caso. En él, se expone el procedimiento cognitivo-conductual aplicado, principalmente, a la ideación delirante perturbadora de un sujeto esquizofrénico con sintomatologia psicótica resistente a la medicación. Un objetivo previo a todo el proceso consistió en establecer una relación de confianza con el paciente. A través de ella, le pudimos sensibilizar para participar en un programa de intervención psicosocial integral, que aliviase sus angustias y le acercase a un estilo de vida alternativo al que hasta entonces habia mantenido.

\section{MÉTODO \\ Sujeto}

Varón de 35 años de edad, derivado a la Unidad de Rehabilitación de Salud Mental "Virgen del Rocio" (Sevilla) en marzo de 1991, por su Equipo de Salud Mental de Distrito (ESMD). Diagnosticado de esquizofrenia tipo paranoide, de 18 años de evolución, según criterios del DSM-III-R (American Psychiatric Association, 1987).

El informe de derivación de su psiquiatra señalaba que "...en la sintomatología actual, predomina su aislamiento social, con una vida en clausura..."; "...ideas delirantes autorreferenciales de perjuicio... fenómenos de imposición o influencia de su pensamiento a través de la televisión... fenómenos de robo del pensamiento o de transmisión del mismo...", "...alucinaciones auditivas de contenido cambiante, en las que aparece como amenaza la posibilidad de su crucifixión..."; "Utiliza un lenguaje disgregado, con tendencia a la repetición de los mismos detalles". Asi mismo, se señalaba que la "conciencia de enfermedad" del paciente "es escasa" y que la "interferencia de la psicopatologla activa es notable e impide su incorporación a actividades normalizadas".

\section{Evaluación 1}

a) Entrevistas con los padres del paciente. Permitieron obtener información sobre la historia 
del trastomo: hijo único en familia sin historia de antecedentes psiquiátricos conocidos. Embarazo y parto sin problemas. Desarrollo psicomotor y socioafectivo normales, adaptación escolar y rendimiento académico adecuados, funcionamiento social normal. Durante la adolescencia le fueron observando "más tímido y triste...", aunque mantenia relaciones estables con sus amistades y un buen rendimiento en los estudios, aprobando COU y la Selectividad. Tras ello, ese verano marchó con sus padres a la playa, donde al parecer sufrió un fracaso amoroso. A regreso de esas vacaciones se manifestaron los primeros signos del trastorno ("nuestro hijo decla tener miedo a salir a la calle y se fue aislando en casa, posteriormente empezó a sufrir alucinaciones auditivas..." ). Acudieron a un especialista quien le diagnostico esquizofrenia paranoide e instauró tratamiento medicamentoso. Contaba entonces con 17 años.

Entre los 18 y 20 años, inició y abandonó estudios correspondientes a las carreras de Biología y Bellas Artes, debido a la interferencia que le provocaban las ideas autorreferenciales. Durante este periodo se fue produciendo una perdida total de sus relaciones sociales habituales, manteniéndose aislado en casa. La sin-tomatología psicótica positiva no remitia, exacerbándose en diferentes períodos. Asi, con 24 años el paciente sufrió su primer ingreso hospitalario, de tres meses de duración, donde recibio tratamiento farmacológico y terapia electroconvulsiva. Con 25 y 28 años tuvo dos experiencias ocupacionales, ambas fugaces (pintar decorados para una caseta de la Feria de Abril de Sevilla y realización a través del INEM de encuestas para el censo), abandonándolas a los pocos días debido a su ideación autorreferencial y a no soportar estar en contacto con gente. Posteriormente sufrió tres ingresos más: con 29 af̂os (por intensificación de sintomatología psicótica, de un mes de duración, siendo de nuevo sometido a terapia electroconvulsiva); con 33 años (de dos meses y medio de duración, por abandono de la medicación y descompensación psicótica); y con 34 años (de un mes y medio, por reagudización de los sintomas). Un año después se produjo la derivación a nuestra Unidad de Rehabilitación. Informes psiquiátricos aportados por los padres referian que "...el cuadro nunca remitio totalmente ni siquiera paso momentos en los que la psicopatología activa no interfiriera en un desarrollo de actividades normalizadas...".

b) En entrevistas mantenidas con el paciente, el primer autor, confirmó el diagnóstico de esquizofrenia tipo paranoide según el DSM-IIIR (American Psychiatric Association, 1987). Además, tomando como guia la Historia de Rehabilitación Psicosocial de la Unidad "Virgen del Rocio" (Cuevas y Perona, 1990) se obtuvo la in-formación siguiente: el paciente presentaba una adecuada adherencia al tratamiento neuroléptico y a las citas establecidas por el psiquiatra de su ESMD. Así mismo, era una persona con quien resultaba fácil entablar conversación. Como problemas principales se identificaron los siguientes: relaciones sociales circunscritas a los padres y profesionales de salud mental que le asistian; ausencia de amistades; temor a salir a la calle; mínimos contactos con elexterior y siempre acompañado por sus padres; ausencia de realización de actividades académicas $u$ ocupacionales; inactividad importante, salvo pintar algún cuadro o escuchar música en su casa; presencia de alucinaciones auditivas $\theta$ ideación delirante, lenguaje disgregado; discurso verbal muy relacionado con la temática psicopatológica y con otros aspectos de la en-fermedad; insatisfacción personal ligada a su estilo de vida y expectativas de futuro; desesperanza, tristeza e importantes niveles de ansiedad.

Las alucinaciones auditivas eran fundamentalmente de contenido negativo y perturbador. No las escuchaba con claridad, sino como susurros dificiles de entender. Consistian en voces que le amenazaban o le decian cosas para él desagradables ("tu novia ya no te quiere", "te van a quemar en una hoguera", "te van a crucificar", "eres el demonio", "te van a matar"). También las "ola" a través de la radio y televisión. Le generaban gran ansiedad y las sufria con mucha frecuencia. En otras ocasiones, los contenidos de las voces resultaban agradables para el paciente ("no te preocupes eres Jesucristo", "eres un genio", "eres una gran personalidad").

Las ideas delirantes, pensamientos inusuales y falsas creencias eran múltiples. Los contenidos delirantes eran variados, pudiendo identificarse tanto, ideas de grandeza que emocionalmente le resultaban gratificantes ("soy un 
genio, hago grandes descubrimientos para el progreso de la humanidad, y los puedo transmitir telepáticamente a la Universidades"); como otras que le perturbaban enormemente ("la gente me adivina el pensamiento", "me quieren matar") y que en gran medida coincidian con el contenido alucinatorio.

c) Formulación de una primera hipótesis de trabajo: nos encontrábamos ante una persona que desde hacia dieciocho anos se hallaba en situación de aislamiento $\theta$ inactividad. Esta situación se relacionaba con la intensa y permanente ansiedad asociada a las alteraciones perceptivas e ideas delirantes; ansiedad que resultaba incompatible con un estilo de vida más normalizado (ver figura $n^{\circ} 1$ ). En consecuencia, manejamos la hipótesis de que una disminución (o eliminación) de la sintomatologia psicótica positiva, reduciría el temor a salir de casa y fa-cilitarla el desarrollo de un programa de rehabilitación psicosocial orientado a alcanzar una forma de vida más normalizada. da de explicación (que en nuestro paciente podia ser esta: "me hablan por telepatia", "me quieren matar porque conozco el secreto de la bomba atómica"), explicación que sería anormal, por serto también la experiencia inicial. En cualquier caso, la llegada a una explicación, aunque sea singular, produciría alivio, circunstancia que podría reforzar dicha explicación.

Tomando como referencia la hipotesis de Maher (1988), se estableció como objetivo principal la intervención sobre la sintomatología alucinatoria, pues si resultaba exitosa, cabria esperar una debilitación de los delirios perturbadores más directamente ligados a las experiencias alucinatorias, y en consecuencia, argumentábamos que el paciente se sentiría más seguro y relajado, y por tanto dispuesto a abandonar su enclaustramiento y con posibilidades de entrar en un programa de rehabilitación. Así mismo, en línea con autores que sugieren que las alucinaciones son más probables durante períodos de gran ansiedad o

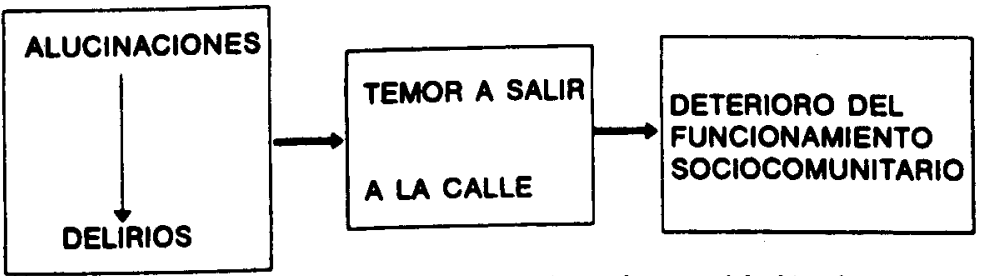

Figura 1.- Formulación de la primera hipótesis

Durante las valoraciones iniciales se habia detectado que la presencia de actividad delirante y alucinatoria era continua. El paciente diariamente "escuchaba voces", sobre todo cuando se encontraba inactivo, es decir durante gran parte del dia, mientras que en aquellos ratos en que se dedicaba a pintar, la frecuencia disminuia o la intensidad se atenuaba. Las alucinaciones frecuentemente coincidian con estados de ansiedad o temor intensos. En un primer momento la sintomatología nos parecio consistente con la hipótesis perceptiva de Maher (1988): una persona delirante presenta problemas perceptivos primarios, de naturaleza fundamentalmente biológica, que ocasionan experiencias anómalas (en ausencia de causa evidente nuestro paciente refería oír voces que le decian "te van a quemar en una hoguera", "te van a matar"); dichas experiencias anómalas producen un sentido de perplejidad, lo cual lleva a su vez a una búsque- estrés (Cooklin, Sturgeon y Leff, 1983), y con el modelo de la discriminación de la realidad (Bentall, 1990), que propone la existencia de distorsiones metacognitivas durante las alteraciones perceptivas; entendimos las alucinaciones auditivas del paciente, como un componente cognitivo de su comportamiento que resultaba de fallos en su habilidad para discriminar entre los eventos imaginados y los del mundo real, y que eran más probables dada la situación de estrés y elevado nivel de activación autonómica en que decia encontrarse de modo habitual.

Por ello, fue seleccionada una técnica de desensibilización a los contenidos de las alucinaciones auditivas. El objetivo era reducir el impacto emocional producido por las voces. De esa manera, decrecería el nivel habitual de activación autonómica del paciente, y con ello la probabilidad de aparición de dichas alteraciones perceptivas. Secundariamente y subordinado al éxito de este tratamiento esperá- 
bamos una reducción en la actividad delirante. Otra posibilidad terapéutica, menos ambiciosa, consistiria en conseguir la modificación de la valencia de las alucinaciones, pasando de contenidos amenazantes a otros de carácter más neutro y desprovistos de perturbación emocional (Siegel, 1975).

\section{Procedimiento 1}

Para el desarrollo de la desensibilización, se ideó la producción de una estimulación auditiva lo más parecida posible a las alucinaciones que sufria el sujeto. Se efectuó una grabación magnetofónica, de unos 50 minutos de duración, tratando de reproducir lo más fielmente posible, sólo las frases de carácter aversivo que el paciente decia escuchar ..."no muy claramente ...como entre susurros o murmullos...". Concretamente se grabaron murmullos, de entre los cuales de vez en cuando surgian con la inteligibilidad requerida frases del tipo... "tu novia ya no te quiere", "te van a quemar en una hoguera", "te van a crucificar", "eres el demonio", "te van a matar". Para ello fue necesaria su colaboración y asesoramiento. Dado que el paciente informó que todos los contenidos alucinatorios le afectaban por igual, no se realizó ninguna jerarqula de frases. Fueron precisas varias grabaciones hasta informó que ..."prácticamente asi es como las escucho".

Previo a las sesiones de desensibilización fue entrenado en la realización de técnicas de relajación muscular. El procedimiento terapéutico fue el habitual en la aplicación de las técnicas de desensibilización. Primero, el paciente se relajaba en un sillón y posteriormente a través de unos auriculares se ponia en marcha la reproducción de la grabación hasta que informaba sentir ansiedad, en ese momento no se interrumpia la audición de la grabación, sino que se mantenía durante unos segundos más, a continuación deteníamos el magnetófono y le dábamos instrucciones para que centrara su imaginación en escenas tranquilizadoras y en la realización de ejercicios de respiración. La secuencia se repetia sucesivamente. Cada sesión duraba entre 40 y 50 minutos, al término de las mismas se le recomendaba que diariamente realizara en casa los ejercicios de relajación.
Según transcurrieron las sesiones, aumentaba de forma progresiva la duración del tiempo de audición de la grabación en ausencia de ansiedad. Tras pocas sesiones (cuatro en el plazo de dos semanas) escuchaba ininterrumpidamente y sin ninguna molestia, la cinta grabada. Los resultados indicaban que habia aprendido a escuchar la cinta sin experimentar ansiedad, pero no se produjo generalización a las situaciones cotidianas en las que sufrla las alteraciones perceptivas. Informes verbales del paciente referian que ni las alucinaciones reales ni la ansiedad a ellas asociada mejoraron; tampoco lo hicieron las ideas delirantes, pues continuaba afirmando que estaba convencido de que la gente le adivinaba el pensamiento y de que le querían matar; asi mismo, la perturbación en el funcionamiento psicosocial se mantenia. Los objetivos terapéuticos no se habian alcanzado, por lo que siguiendo el proceso de evaluación y modificación de conducta (Fernández Ballesteros y Carrobles. 1981), consideramos la reevaluación del caso y la formulación de otras hipótesis alternativas, explicativas del mantenimiento del problema.

\section{Evaluación 2 (reevaluación del caso)}

a) Entrevistas con el paciente. Información verbal aportada por el sujeto en una serie de entrevistas centradas en el análisis funcional secuencial (Fernández Ballesteros y Carrobles, 1981; Segura, Sánchez y Barbado, 1991) de las ocurrencias más recientes de su sintomatologia psicótica, posibilitó identificar a determinadas ideas delirantes como las variables a modificar. Asi, el análisis secuencial parecia señalar que ciertas ideas funcionaban a modo de antecedentes de las alucinaciones auditivas. Se observó que la actividad delirante general del sujeto era prácticamente continua, aunque no siempre iba seguida de actividad alucinatoria. De hecho, otras ideas (delirios de grandeza y falsas creencias) no implicaban perturbación emocional, mientras que aquellas ideas cuyo contenido era negativo en relación al paciente, sí elicitaban ansiedad, y cuando ésta era muy elevada entonces aparecian las "voces" (que en contenido "coincidian" con el de algunas de las ideas delirantes más perturbadoras). Las ideas delirantes no estaban especialmente ligadas a situaciones especificas potencialmen- 
te elicitadoras de ellas, al contrario, podian ocurrir en cualquier momento o lugar, y podian entenderse como fruto de la interacción del sujeto consigo mismo.

b) Segunda hipótesis. La nueva evaluación sugeria que de las múltiples ideas delirantes, sólo debian ser objeto de tratamiento las de contenido perturbador para el sujeto; es decir. las que le provocaban temor, probabilizaban la aparición de alucinaciones y se relacionaban funcionalmente con un desempeno psicosocial pobre. Este tipo de ideas consistian en creer que gente indeterminada quería acabar con su vida violentamente debido a que poseía el secreto de cómo construir la bomba atómica. Además, creía que algunas personas podian conocer sus pensamientos, por ello era más seguro permanecer en casa. La antigüledad de estas ideas coincidia con la historia del trastorno, dieciocho años. En la evaluación (ver figura n'2) se identificaron como posibles variables relacionadas con el mantenimiento de las ideas delirantes, y por consiguiente objeto de modificación, las siguientes: 1) Principalmente, la aceptación acritica de sus creencias, con falta absoluta para generar otras explicaciones alternativas de carácter lógico y empírico, circunstancia que podla favorecer el mantenimiento de los delirios. $Y$ de modo secundario, 2) La atención que durante años habia recibido por parte de su familia y profesionales de salud mental. Por un lado, la constante preocupación de los padres ante el sufrimiento del paciente, y por otro, la atención de los profesionales hacia cuestiones principalmente clinicas, podian considerarse elementos con función de refuerzo en relación a las quejas delirantes del paciente. 3) El aislamiento casi absoluto, situación que lejos de facilitar la obtención de pruebas de realidad contrarias a sus creencias de perjuicio, favorecia su mantenimiento. Así, el progresivo alejamiento social al principio del trastorno y el posterior desarrollo de un patrón de comportamiento evitativo aislándose en casa, constituyeron un estilo de vida desarrollado como consecuencia del malestar producido por la sintomatologia psicótica y cuya funcionalidad consistla en evitar la experimentación de mayores tasas de ansiedad ante la "posibilidad" de que si salia a la calle "podrian acabar con su vida". 4) La ausencia de actividades sociocomunitarias. La no realización de actividades ocupacionales en las que centrar su atención favorecian que ésta se mantuviera continuamente dedicada a sus fantasías. 5) Las alucinaciones auditivas amenazantes, que suponian un continuo "recuerdo" que le reafirmaba en sus temores.

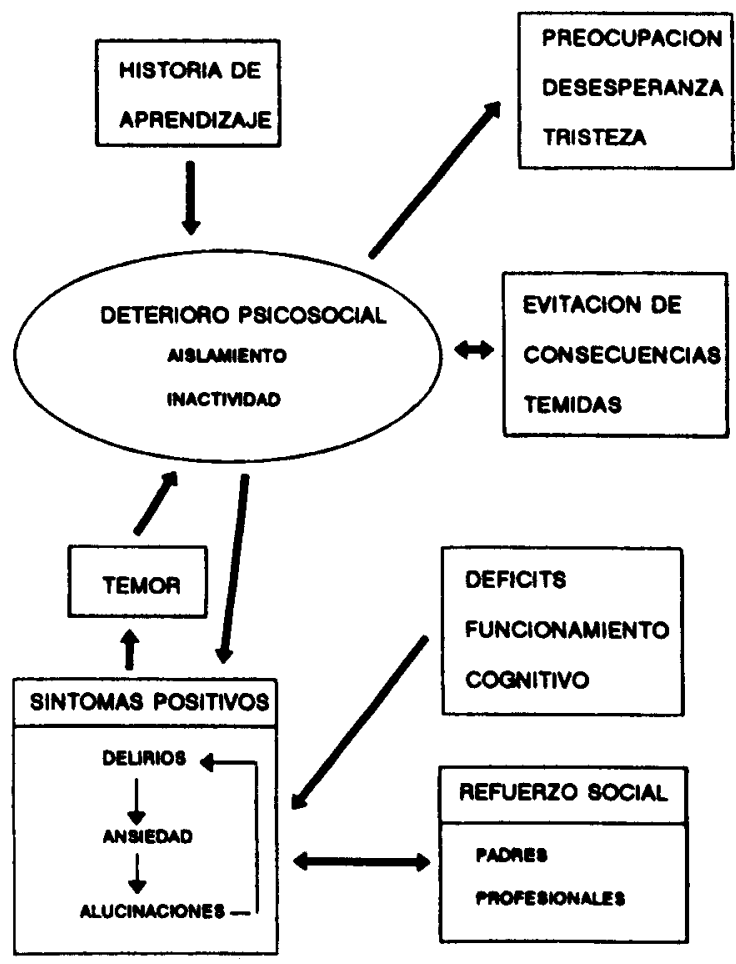

Figura 2.-Formulación de la segunda hipótesis

La nueva hipótesis de trabajo predijo que de la modificación del grado de convicción del paciente en sus creencias perturbadoras, derivaria una reducción importante de sus temores y una disminución en la actividad alucinatoria de contenido consistente con dichas creencias delirantes. En consecuencia, estaria en una situación más favorable para abandonar el aislamiento e integrarse en actividades sociocomunitarias.

c) Medidas e instrumentos: 1) Escala de valoración psiquiátrica, BPRS (Brief Psychiatric Rating Scale; versión adaptada por Lukoff, Nuechterlein y Ventura, 1986). Concretamente, en base al autoinforme del paciente, se valoraron cinco de sus itemes: ansiedad, suspicacia, contenidos inusuales del pensamiento, grandiosidad y alucinaciones. Se tomaron, una medida antes del tratamiento, una al final de la intervención sobre los delirios, y seis durante el 
seguimiento (al 1, 6, 12, 24, 36 y 48 meses). 2) Valoración del grado de convicción en sus ideas, mediante una escala subjetiva cuyos valores se extendian a lo largo de un continuo, desde $0 \%$ ("no 10 creo en absoluto"), hasta 100\% ("estoy absolutamente convencido"). Se tomaron medidas en doce sesiones de línea base y durante las dieciocho sesiones de tratamiento de los delirios. Posteriormente, se repitieron las medidas en siete sesiones de seguimiento al $1,3,6$, $12,24,36$ y 48 meses. 3) Escala de habilidades para la vida independiente, ILSS (Independent Living Skills Training; Wallace, Boone, Donahoe y Foy, 1985). Se utilizaron cuatro de sus subtests: apariencia y cuidado personal, capacidad para el manejo del dinero, uso de transportes y realización de actividades socio-ocupacionales, los cuales fueron administrados antes del tratamiento, al terminar el tratamiento y a los 24 meses de seguimiento. 4) Observación y registro de la tasa de verbalizaciones relacionadas con las ideas delirantes objeto de tratamiento. Las observaciones abarcaban períodos de una semana, obteniéndose posteriormente, la media diaria de tales verbalizaciones. Se realizaron antes del tratamiento, después del tratamiento, y al 1 , $3,6,12,24,36$ y 48 meses de seguimiento. Estas observaciones fueron llevadas a cabo en dos contextos: en casa por los padres, y por los monitores en los talleres ocupacionales en que participó. En ambos casos se registraban las verbalizaciones delirantes que de modo espontáneo manifestaba el paciente.

\section{Procedimiento 2}

Basándonos en la segunda y definitiva hipótesis formulada, los objetivos de intervención se centraron en: a) debilitar el grado de convicción del paciente respecto a los delirios que le provocaban más ansiedad e interferencia, así como mayor probabilidad de alucinar; b) Intervenir sobre las verbalizaciones delirantes del paciente. Previo al inicio del tratamiento, se aprovecharon las doce entrevistas de linea base, para establecer una relación empática con el paciente, el cual aceptó el tratamiento con la intención de aliviar la perturbación emocional que sufria.

a) Tratamiento de las ideas delirantes: Las creencias seleccionadas para tratamiento, fueron las siguientes: 1) "conozco el secreto sobre cómo construir la bomba atómica", 2) "si la gente se entera de ello me matarán", 3) "algunas personas pueden leerme el pensamiento". Cada creencia fue anotada con las mismas palabras empleadas por el paciente (BrettJones, Garety y Hemsley, 1987). La idea que más temor le provocaba era la segunda ("si se enteran me matarán"), la referente a las consecuencias negativas que de su "conocimiento" podria derivarse si alguien se enterase de ello, circunstancia "probable", al creer también que le podian leer el pensamiento. Además, de modo arbitrario, decidimos controlar una cuarta creencia: “...Dios nos pone los sueños en la mente por las noches... ya que soñamos cosas que nunca hemos vivido, ni siquiera imaginado"; esta creencia era emocionalmente neutra, no resultando perturbadora para el paciente y no iba a ser objeto de tratamiento.

Durante unos dos meses, se llevaron a cabo 18 sesiones de intervención. Cada sesión duraba entre 40 y 60 minutos. En primer lugar y desde un enfoque psicoeducativo,se le explico: a) la relación que existe entre cognición, emoción y comportamiento (Beck, 1979); b) el papel de las cogniciones como elementos que a modo de hipótesis intentan explicar y valorar la realidad, explicaciones que pueden ser más o menos veraces, aunque no necesariamente certeras ni únicas; c) la importancia de las evidencias empíricas como elementos que pueden incrementar la probabilidad de certeza de una hipótesis. Todo ello se realizó en un contexto relajado en el que el terapeuta (primer autor) hacia reflexionar al paciente, apoyándose para ello en la utilización del diálogo socrático y en ejemplos ajenos a la problemática del paciente.

El siguiente paso consistio en solicitar al paciente que aportara evidencias sobre sus creencias, para ello el terapeuta formulaba preguntas del tipo: ..." ¿qué te hace pensar que sabes hacer la bomba atómica?", o..." ¿qué hechos te hacen pensar que la gente te quiere dañar?". Como respuesta, el paciente aportaba pobres especulaciones, razones absurdas e inconsistentes, imaginaciones carentes de base real, tanto sobre sus conocimientos en fisica y química, como sobre el peligro que por ello corria. A partir de ahi, el terapeuta presentaba más preguntas: ..." ¿son suficientes tus conocimientos a nivel de COU para poder construir una bomba atómica?", ..."¿cualquier persona que haya realizado 
estudios similares a los tuyos, puede también hacerla?", ..." ¿es suficiente haber estudiado la carrera de fisica o de química parar construirla o seria necesario poseer más conocimientos y recursos... ¿cuáles?... ¿los tienes tú?". Las respuestas del paciente se analizaban conjuntamente, señalando los aspectos a favor $y$ en contra. Asi mismo, el terapeuta le aportaba para su análisis "creencias erróneas", de contenido diferente a las suyas aunque equivalentes: ..." ¿qué pensarias si te digo que tengo suficientes conocimientos para acabar con las enfermedades, y lo justifico diciendo que eso se conseguiria elaborando "fármacos" muy potentes?", ..." ¿o si te digo que sé realizar un trasplante de corazón?... ies muy fácil!, sólo hace falta un buen equipo de profesionales, los instrumentos necesarios, el donante, el receptor y ...suturar los vasos correspondientes!". El paciente rechazaba tales creencias, señalando la necesidad de estudiar medicina, especializarse en cirugía cardiovascular, de aprender y practicar, etcétera; a continuación comparábamos estos ejemplos con sus creencias y argumentos. A medida que avanzaban las sesiones, el paciente iba contemplando la posibilidad de que sus conocimientos eran insuficientes y que en realidad no posela una teoria basada en hechos; carecia de formación, recursos y experimentación... en definitiva, sus ideas eran simples especulaciones.

Posteriormente, se le planteó que quizás existieran otras alternativas a las percepciones y explicaciones que él tenía sobre la realidad. Se le animo a explorarlas. Pero no fue capaz, evidenciando un déficit en la generación de alter-nativas, por lo que fue el propio terapeuta quien le presentó otra posibilidad: "...téricamente, una hipotesis alternativa a tu creencia de que te quieren matar porque sabes el secreto de la bomba atómica... podría ser sencillamente la contraria...".

Respecto a su principal temor, ser asesinado, se le planteaban nuevas cuestiones: ..." $i$ alguien te está tratando mal?... ¿te han agredido alguna vez"?, ..." ¿alguna persona te ha amenazado verbalmente... o te ha dicho que te quiere matar por tus conocimientos?"... ante las negativas del paciente, el terapeuta continuaba... "dices que desde hace muchos años te quieren asesinar... ¿cómo te explicas entonces que cuando sales a la calle, cuando te desplazas hasta la Unidad de Rehabilitacion, nadie te haga daño?"..." ¿no te parece que si alguien hubiese querido hacerte daño, durante estos dieciocho años, ya habria tenido tiempo suficiente para intentarto... cómo te explicas que no haya sido asi?" ... ¿ ¿no es evidencia de lo contrario... de que la gente no te quiere dañar?".

Otra linea de trabajo consistió en cuestionar la relación que establecia entre poseer determinados conocimientos y peligrar por ello su vida. El terapeuta le planteaba: "¿por qué razones poseer un conocimiento ("hacer una bomba") habria de significar consecuencias negativas para ti?... ¿qué evidencias existen de que se esté castigando, crucificando, omatando a la gente por tener conocimientos?... ¿está considerado como falta o pecado en algún código ético o moral?... ¿está tipificado cómo delito?"...

Durante las 18 sesiones insistiamos sobre los contenidos expuestos. Progresivamente el paciente fue verbalizando encontrarse menos ansioso en su vida cotidiana y en las entrevistas iba llegando a conclusiones más realistas: ... "los únicos conocimientos que yo tengo sobre fisica y química son mis recuerdos de bachillerato $y$ eso es insuficiente para construir una bomba"... concretamente este razonamiento hacla que disminuyera su preocupación ante la posibilidad de que alguien le pudiera leer el pensamiento. También se fue debilitando su convicción respecto a la relación que establecia entre poseer conocimientos y sufrir consecuencias peligrosas, llegó a la conclusión de que en realidad nadie queria dañarle; asi mismo empezó a cuestionar, espontáneamente, la posibilidad de lectura del pensamiento, razón por la que esta idea no llegó a ser trabajada directamente. Al final de estas sesiones el paciente no habla llegado a un grado de convicción en sus ideas igual a cero, pero si se habla producido un debilitamiento muy notable, asi como cambios positivos en su actitud y relación con el mundo exterior. Ese fue el momento en que decidimos dar paso a la fase de seguimiento.

b) Intervenciones sobre las verbalizaciones delirantes del paciente. La conducta verbal del paciente se caracterizaba por unos contenidos muy relacionados con sus ideas delirantes, resultando significativa la escasez de contenidos 
conversacionales socialmente adecuados. Ello era consistente con su larga historia de ausencia de relaciones sociales normalizadas y con la continua presencia de preocupaciones, temores y sintomas psicóticos. Tanto en las interacciones con sus padres como con los profesionales del taller ocupacional de nuestra Unidad, cuando empezó a asistir, eran frecuentes verbalizaciones del tipo "¿me puedes leer el pensamiento?", "la gente me quiere hacer daño", "¿crees que me van a hacer daño?", "me van a matar", "me hablan a través de la radio", "van a crucificarme". También eran con frecuencia tema de conversación, aquelios relacionados con aspectos de la enfermedad, como por ejemplo, hablar sobre fármacos, psiquiatras o centros de salud mental. Para controlar la evolución en la tasa de este tipo de conductas se intervino a dos niveles: en casa y en los talleres ocupacionales en que participó.

A los padres, en primer lugar se les ofrecio información y justificación sobre el tratamiento indicado. Posteriormente, se les enseñó cómo observar y registrar el número de verbalizaciones delirantes que su hijo, como era habitual, les dirigía. No tratándose de tomar nota de cualquier comentario inusual, sino sólo de aquellos que habiamos identificado como perturbadores para el paciente. Para facilitar el aprendizaje dedicamos dos sesiones de entrenamiento, practicando mediante ensayos de conducta, ejemplos sobre qué y cuándo registrar. Complementariamente, se les instruyó y adiestró en el uso de los principios del condicionamiento operante con un objetivo principal: minimizar la atención dispensada a las verbalizaciones delirantes, con la intención de disminuir su tasa; secundariamente, se intentaría potenciar el mantenimiento de conversaciones socialmente más adecuadas. El primer autor y un ayudante, dedicaron tres sesiones de entrenamiento (modelado, ensayos de conducta y retroalimen-tación) para facilitar el desarrollo de tales habilidades en los padres del sujeto.

En los distintos talleres ocupacionales en que participo, las indicaciones para los monitores fueron en el mismo sentido que las señaladas a los padres del paciente. No obstante, en estos contextos, dada la experiencia de estos profesionales, no fue necesario llevar a cabo sesiones de entrenamiento.

\section{RESULTADOS}

a) Valoración psiquiátrica de la ansiedad, suspicacia, contenidos inusuales del pensamiento, grandiosidad y alucinaciones del paciente, a través de la escala BPRS (Lukoff et al. 1986).

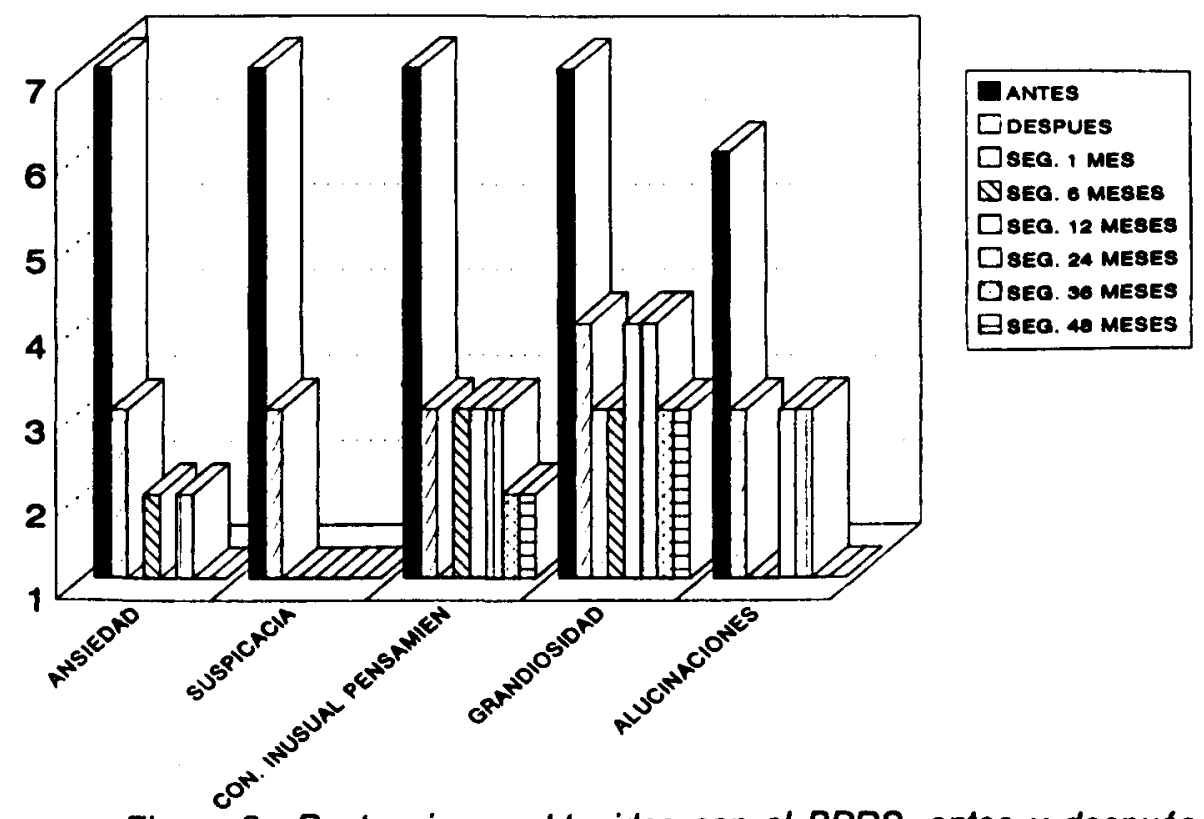

Figura 3.- Puntuaciones obtenidas con el BPRS, antes y después del tratamiento $y$ en los períodos de seguimiento 
Figura 4

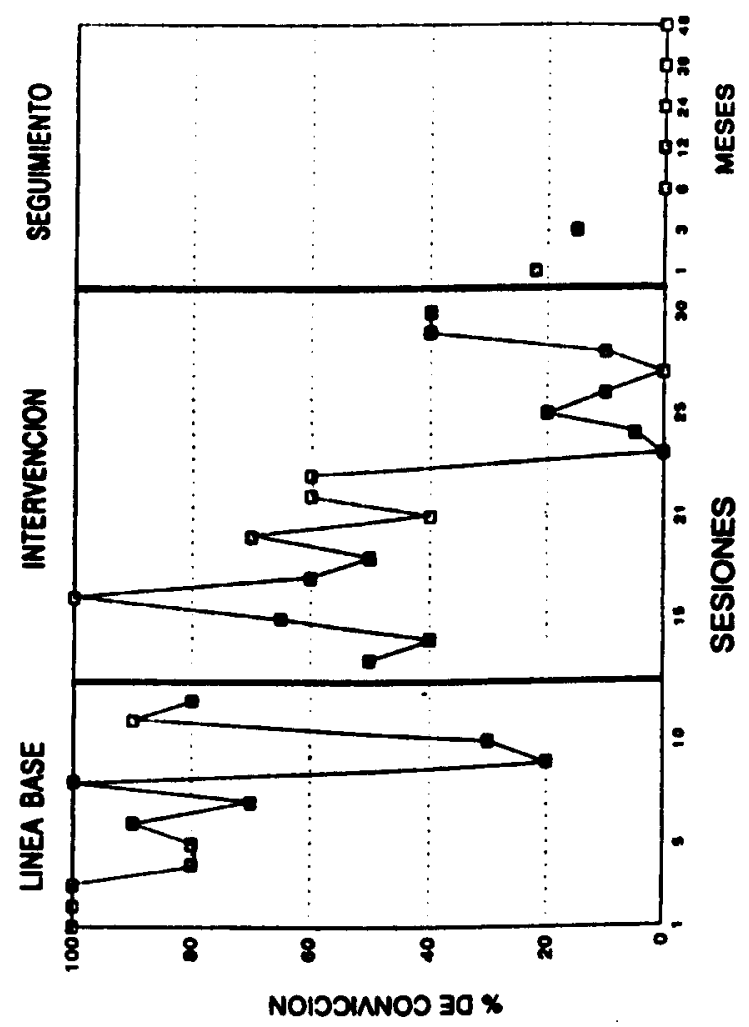

Figura 6

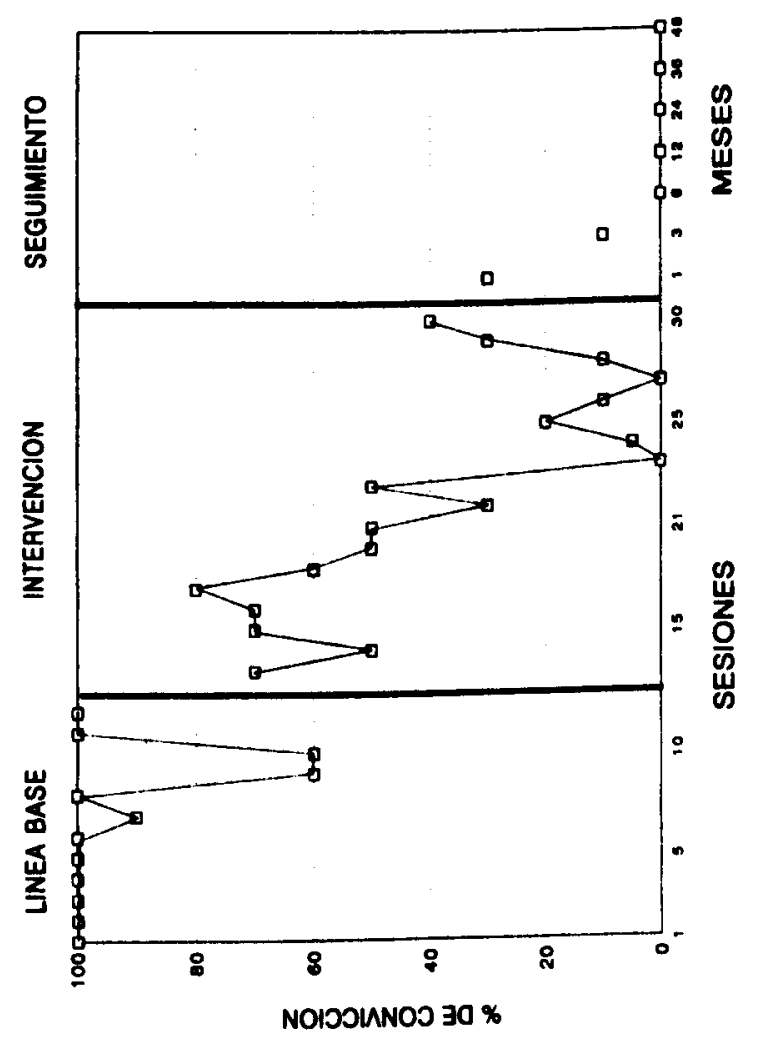

Figura 5

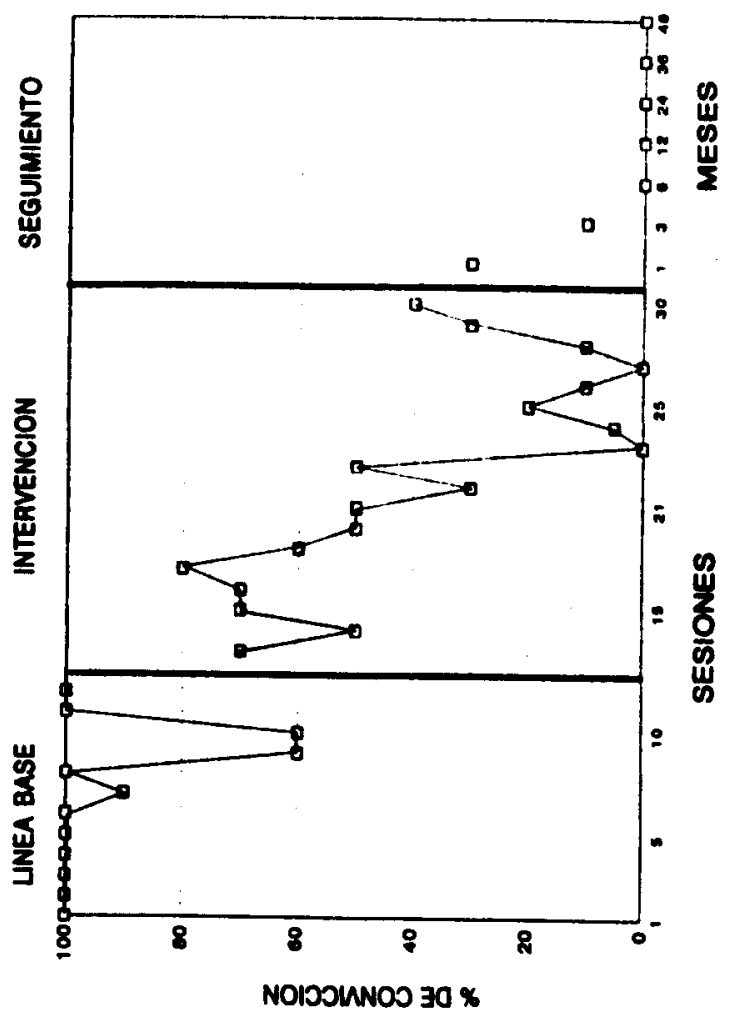

Figura 7

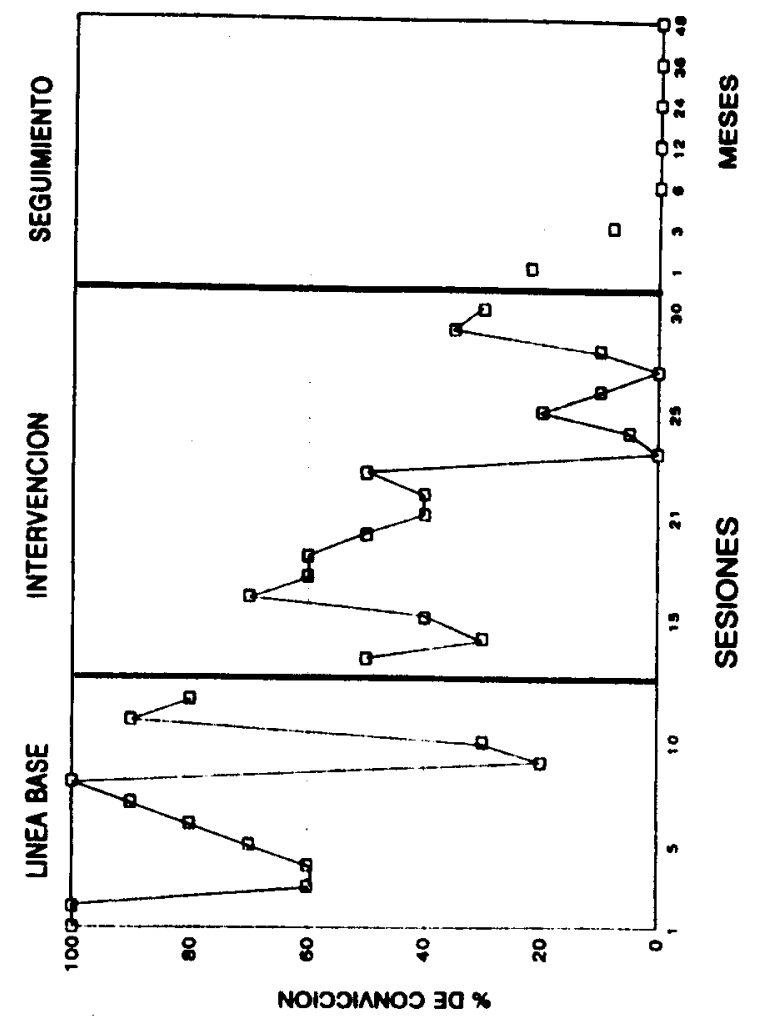


Las respuestas del paciente se sitúan a lo largo de una escala ordinal de 1 a 7 puntos. Valoraciones superiores a los 3 puntos se consideran de intensidad patológica. En la figura $n^{\circ} 3$, puede observarse cómo antes del tratamiento, la sintomatologia alcanzaba valores de intensidad máximos, con el consiguiente grado de preocupación e interferencia en el funcionamiento. En la medida postratamiento, ya se aprecian cambios notables. Excepto el ítem grandiosidad, el resto alcanzaba puntuaciones de intensidad no patológica. Por último, en la fase de seguimiento se constata una práctica desaparición de ansiedad, suspicacia $y$ alucinaciones, mientras grandiosidad y contenidos inusuales del pensamiento se mantienen dentro de unos limites entre leve y moderado, pero en cualquier caso sin afectación en el funcionamiento.

b) Grado de convicción en las ideas delirantes. Al termino de la última sesión de tratamiento los porcentajes de convicción en las cuatro ideas no habian llegado al $0 \%$, pero evolucionaban hacia esa cifra. Es decir, se produjo una progresiva y notable disminución en la fuerza de convicción del paciente en sus ideas y también en la ansiedad que le provocaban. Además, empezaron a disminuir, llegando a desaparecer, las alucinaciones auditivas (cuyos contenidos coin- cidian con sus ideas de que le matarian). Todo ello permitió desbloquear la situación de aislamiento $e$ inactividad y fue punto de partida de una evolución más positiva, con la desaparición de muchas de sus dificultades y con el desarrollo de un funcionamiento social y ocupacional más normalizado: el paciente participó en actividades de rehabilitación ocupacional en el taller de nuestra Unidad, se integró en cursos de cerámica y de encuadernación en centros ocupacionales comunitarios, y desarrollo relaciones sociales estables con otros pacientes. Tras el tratamiento se llevó a cabo un seguimiento de cuatro años de duración. En las dis-tintas evaluaciones llevadas a cabo se mantenian los resultados alcanzados mediante el tratamiento psicológico. Las figuras $n^{\circ} 4$, 5,6 y 7 , muestran la evolución, a lo largo de las distintas fases del proceso, de los porcentajes sobre el grado de convicción en cada una de las ideas.

c) Valoración del funcionamiento socio-comunitario. La figura $n^{\circ} 8$ representa, en porcentajes, datos sobre el funcionamiento social del paciente (ILSS, Wallace et al. 1985), antes del tratamiento y después, observándose una franca mejoria. En el seguimiento efectuado a los 24 meses se mantenian los resultados alcanzados.

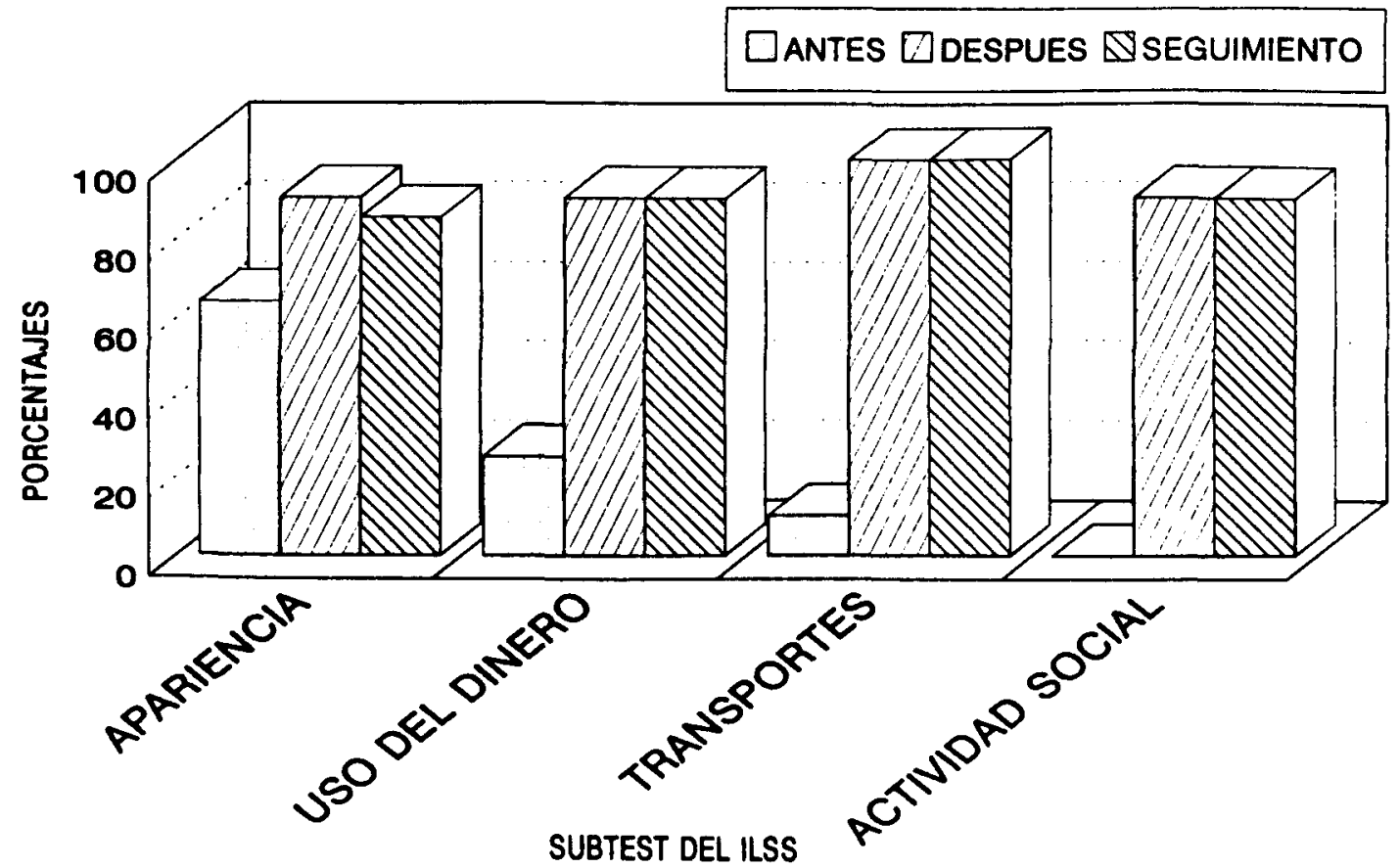

Figura 8.- Nivel de autonomia según el ILSS, antes y después de la intervención y en el seguimiento 


\section{PROMEDIO VERBALIZACIONES/DIA}

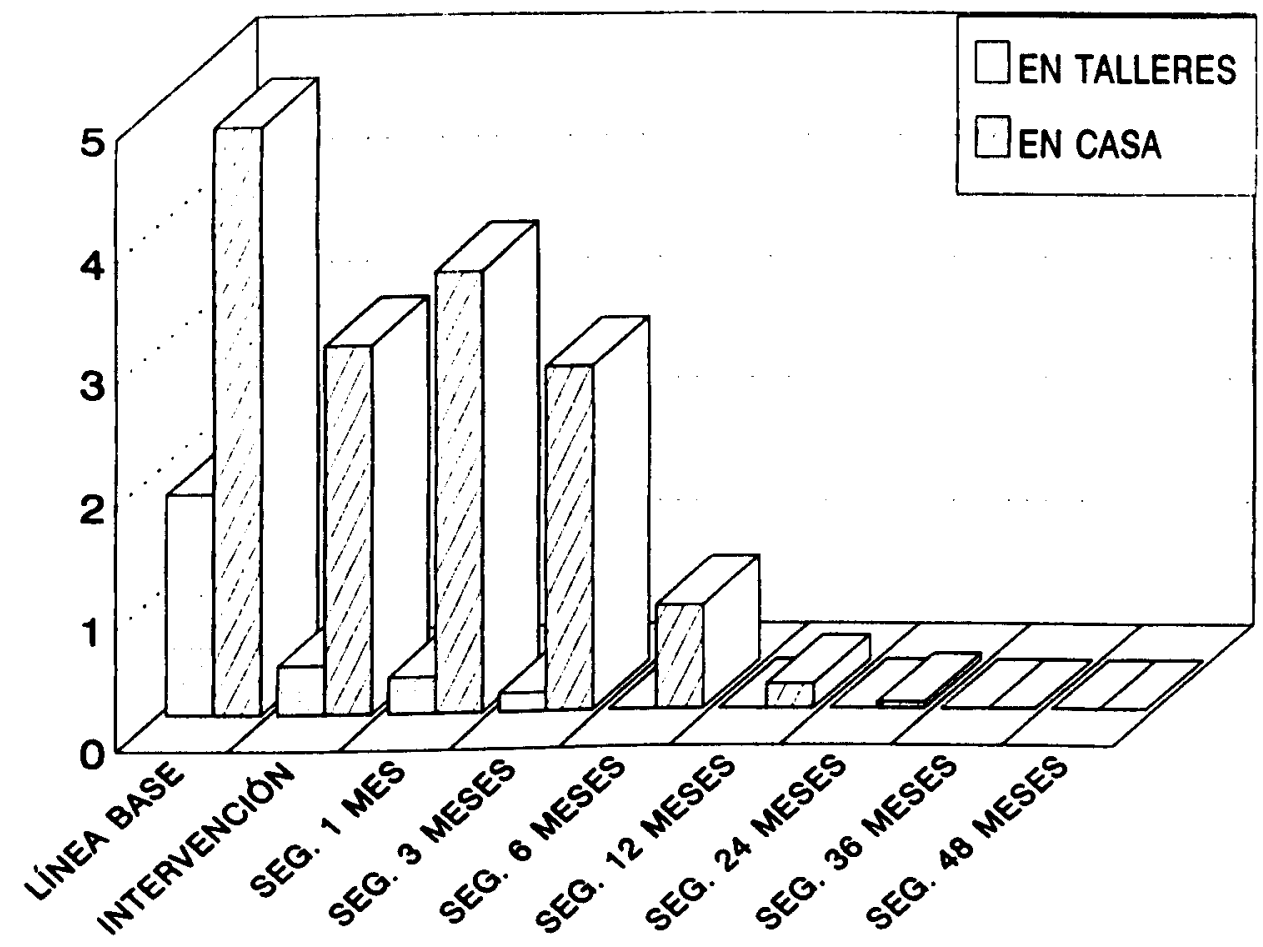

Figura 9.- Promedio de verbalizaciones delirantes al dia, durante las fases de linea base, intervención y seguimiento, en el taller ocupacional de la Unidad de Rehabilitación y en su casa

d) Tasa de verbalizaciones delirantes. La figura $n^{\circ} 9$, refieja los resultados correspondientes a la media diaria de las verbalizaciones delirantes "diana", manifestadas en los diferentes talleres ocupacionales en que participó y en casa. En dichos contextos, estas verbalizaciones disminuyeron primero y desaparecieron después. Durante los cuatro años de seguimiento los resultados se mantuvieron estables.

\section{DISCUSIÓN Y CONCLUSIONES}

La consideración de los cambios producidos en la sintomatología psicótica y en el estilo de vida de una persona que llevaba dieciocho años en situación de aislamiento social, sugiere la eficacia de las aportaciones cognitivoconductuales en el tratamiento de los delirios.

No obstante, al tratarse de un estudio carente de mayor control experimental, las conclusiones respecto a la eficacia de la intervención han de ser tomadas con suficiente precaución. Pues si bien el tratamiento se centró en las ideas delirantes perturbadoras, resulta dificil determinar si otras variables pudieron contribuir a la modificación conductual de nuestro paciente, asi como su posible peso especifico. En este sentido, una primera cuestión importante fue que para poder realizar el tratamiento de las creencias, el paciente tenla que desplazarse a nuestra Unidad, ello suponia salir de casa, exponiéndose de modo repetido a una situación temida y en la que además, realmente no tenía lugar la materialización de sus temores; ello, obviamente, implicaba un proceso de exposición en vivo y la presencia de pruebas de realidad contrarias a sus creencias, elemento éste que durante la terapia seria utilizado por el terapeuta. Segundo, al poco tiempo el paciente inició su asistencia al taller ocupacional de nuestro dispositivo, donde le fue posible centrar su atención en actividades y en relaciones sociales, reales y gratificantes, alternativas a las cogniciones delirantes y alucinatorias en las que habitualmente se centraba. En tercer lugar, otro aspecto importante, fue el cambio 
operado en la actitud de los padres; como ya explicamos, éstos trataron de desarrollar conversaciones de contenido normalizado y de disminuir la atención a las verbalizaciones delirantes; esta actitud que también asumieron los monitores del taller ocupacional pudo, desde un punto de vista operante, incidir en el debilitamiento de las mismas.

En cualquier caso, los resultados obtenidos en el estudio no contradijeron nuestra segunda hipótesis, la cual pronosticaba que un debilitamiento en el grado de convicción del paciente respecto a sus creencias delirantes iría seguido de una disminución de sus miedos (Chadwick y Lowe, 1994), alucinaciones auditivas y aislamiento social, posibilitando un mejor funcionamiento sociocomunitario. A su vez, la integración en actividades ocupacionales y el establecimiento de relaciones sociales con otros enfermos, fue importante para consolidar la eliminación de sus delirios, pues suponia continuas pruebas de realidad, contrarias a los mismos.

Si bien, el enfoque cognitivo-conductual de los delirios señala la posibilidad de trabajar a dos niveles: inferencias delirantes y cogniciones evaluativas subyacentes (Chadwick et al. 1996); en nuestro estudio, el trabajo que se limito a las primeras, permitió la obtención de resultados positivos.

Respecto a la tercera creencia: "algunas personas pueden leerme el pensamiento", hay que decir que su carácter aversivo estaba estrechamente ligado al temor que le producía la idea de poder ser asesinado por sus conocimientos, de tal forma, que cuando el grado de convicción en estas creencias decreció y desapareció la ansiedad concomitante, el paciente de modo "lógico" comenzó a despreocuparse ("si la gente no quiere ni tiene motivos para dañarme... no me importa que puedan leerme el pensamiento"), lo cual es comprensible considerando las relaciones existentes entre dicho trio de ideas. Posteriormente y de forma espontánea fue desechando la posibilidad de lectura del pensamiento ("no es posible leer a alguien su pensamiento"). Hubo una cuarta creencia emocionalmente neutra que tampoco fue tratada: "Dios nos pone los sueños en la mente por las noches..."; aproximadamente a mitad del proceso de intervención, modificó su creencia y aportó espontáneamente otra alternativa: "hay teorias psicológicas que explican la formación de los sueños". En línea con Lowe y Chadwick (1990), estos casos podrían interpretarse como una generalización del tratamiento a otras creencias y significar que el paciente adquirió nuevas habilidades para pensar más racionalmente.

De todas formas, seria legitimo dudar que los resultados del tratamiento psicológico de determinadas ideas delirantes deban de generalizarse a cualquier otra no tratada. En este sentido, hay que considerar que no todas las ideas delirantes son de la misma naturaleza ni comparten una misma funcionalidad. En nuestro estudio fueron tratadas ideas con un fuerte componente aversivo para el paciente, pero también existian otras creencias, no tratadas, que para él significaban fuente de gratificación y elevaban su autoestima ("soy el mejor pintor del mundo"), e incluso otras desprovistas de carga emocional. Así, hay que destacar que en las dos exacerbaciones sintomatológicas ocurridas durante el largo período de seguimiento, transitoriamente volvió a creer que sus ideas podian ser leídas, pero curiosamente sólo aquellas que le resultaban gratificantes, concretamente ideas de grandeza ("asi puedo transmitir mi saber sobre el arte de la pintura"), ideas que obviamente tenian una funcionalidad distinta produciendo un efecto positivo en su autoestima. En cambio, continuaba sin creer que pudieran ser "leidas" otras de sus ideas, especialmente aquellas cuyo conocimiento supusiera posibles consecuencias aversivas para él, o las que no produjeran admiración en otras personas. Esto sugiere que no habria que esperar necesariamente la generalización de los efectos del tratamiento a todas las ideas delirantes de similar topografia, a modo de fenómenos discretos (existencia de lectura de pensamiento versus ausencia, se delira versus no se delira), sino a aquellas que compartieran una misma funcionalidad. Y concretamente, la idea de lectura del pensamiento en relación a contenidos de grandeza cumplia una función muy diferente, función que en ningún momento fue motivo de modificación, ni directa ni indirectamente. Ideas con distinta funcionalidad no deben ser sensibles a una misma intervención. ni necesariamente ser objeto de tratamiento. De forma similar, hay que destacar que cuando durante el periodo de seguimiento el paciente sufría ocasionales alucinaciones auditivas, és- 
tas eran congruentes con las ideas de grandeza ("debes pintar el Vaticano") y emocionalmente gratificantes.

Los diferentes valores observados en el grado de convicción respecto a las cuatro creencias controladas, es consistente con el punto de vista que concibe a los delirios como las partes más extremas de diferentes dimensiones en las que se pueden estudiar las creencias y los juicios (Baños y Belloch, 1995). Así, en el presente estudio las ideas delirantes no se mantuvieron inmodificables a lo largo del tiempo, debilitándose de forma progresiva el grado de convencimiento del sujeto.

Como sabemos, las expectativas del experimentador ante un determinado tratamiento puede ser una variable que incida en que el sujeto responda en la dirección deseada. Por ello, además de la valoración sobre el grado de convicción, es importante la obtención de otras medidas que ratifiquen la fiabilidad de los datos obtenidos en las sesiones individuales con el paciente. Estas medidas deben ser consistentes con la hipotesis de trabajo. Asl en nuestro estudio, si realmente se estaba produciendo un debilitamiento en las convicciones, era de esperar que ello se acompañase de un decremento en la manifestación de verbalizaciones sobre preocupaciones y miedos en la casa y en los contextos ocupacionales, asi como de una mejoria en su funcionamiento social, cosa que ocurrio.

Un aspecto importante de este estudio es el largo seguimiento efectuado. El hecho de que una serie de ideas perturbadoras que el paciente habia sufrido durante dieciocho ańos. continuaran desaparecidas a los cuatro años de seguimiento, refuerza la idea de que el tratamiento fue efectivo. Como ya hemos mencionado, durante los cuatro anos de seguimiento se produjeron dos exacerbaciones sintomatologicas, resultando significativo que en ellas no reaparecieran ni las ideas tratadas ni las alucinaciones auditivas amenazantes; además, el paciente no requirió ser hospitalizado y no interrumpió sus actividades ocupacionales.

Como consecuencia de los resultados de la primera intervención, la desensibilización a los contenidos alucinatorios, fue preciso replantear el caso. Sabemos que son muchas las variables que intervienen en un proceso de intervención que pueden escapar al control del expe- rimentador: variables relacionadas con el terapeuta y con el paciente, con la evaluación, con la elección de la técnica de intervención y su aplicación, y con posibles variables contaminadoras (Fernández Ballesteros y Carrobles, 1981). Asi por ejemplo, podemos señalar que la ausencia de generalización de los resultados obtenidos en la situación de desensibilización pudo estar relacionada con la utilización de una estimulación auditiva próxima a la real pero no idéntica. Por tanto no se puede afirmar que nuestra primera hipótesis, basada en el modelo perceptivo de Maher (1988) fuera errónea. Toda vez que el tratamiento no logró eliminar las alucinaciones, nada puede inferirse sobre la ausencia de debilitamiento de las ideas delirantes.

El enfoque conductual se centra en la modificación de sintomas y conductas, pero considerando no sólo sus aspectos topográficos sino desde un punto de vista funcional. Las intervenciones deben favorecer el funcionamiento del sujeto en su propio entorno. Para ello es preciso integrar toda la problemática clínica y psicosocial dentro de una única hipótesis explicativa, a partir de la cual y de forma coherente se determinarán las intervenciones de elección. AsI mismo, el análisis funcional permite identificar los objetivos clave del tratamiento, es decir aquellos sintomas o conductas que debido a sus interrelaciones funcionales permiten hipotetizar que de su modificación, indirectamente resultarán cambios en otros sintomas (Cuevas y Perona, 1995). En nuestro caso, la problemática era compleja y existian múltiples sintomas y deficiencias en el estilo de vida del paciente, potencialmente susceptibles de intervención directa. No obstante, el análisis funcional de los sintomas permitió formular una segunda hipótesis de trabajo alternativa que identificaba como objetivo clave de tratamiento sólo a determinadas ideas delirantes perturbadoras, ideas que funcionaban a modo de antecedentes y que en conjunción con actividad autonómica elevada, probabilizaban la aparición de alucinaciones auditivas, y dificultaban el funcionamiento social.

El tratamiento psicológico de síntomas esquizofrénicos suscita muchas preguntas, una de ellas se refiere a ¿cuándo dar por finalizado el tratamiento?. En el presente caso es de señalar que al final de la fase de intervención el paciente no habia llegado a un grado de 
convicción en sus ideas igual a cero, pero si se habia logrado un debilitamiento importante en sus creencias, así como modificaciones positivas en su actitud hacia el mundo externo. Ello puede sugerir un criterio en relación a "cuándo" finalizar un tratamiento de este tipo: cuando se logre que el sujeto dude razonablemente de sus ideas y sobre todo cuando ello vaya acompañado de mejoras en su funcionamiento social.

Otra cuestión se refiere a cómo implicar en el tratamiento a pacientes cuyo juicio de realidad se halla comprometido. La propia sintomatología de los pacientes psicóticos, asi como bajos niveles en su conciencia de enfermedad, pueden hacer dificil que entiendan la justificación de determinadas intervenciones. Trabajos como el presente sugieren que no constituye requisito imprescindible, aunque si deseable, la presencia de plena conciencia de enfermedad para el tratamiento cognitivo-conductual de ideas delirantes en esquizofrénicos no hospitalizados. En pacientes de estas caracteristicas puede resultar de interés ofertarles el tratamiento como la posibilidad, por una parte. de aliviar temores, y por otra, de que en el futuro puedan llevar una vida más normal. Este planteamiento puede facilitar la participación y colaboración en terapias psicológicas.

En un campo nuevo como es el tratamiento psicológico de los sintomas psicóticos esquizofrénicos es importante la realización de largos seguimientos que permitan valorar el alcance y duración de estas intervenciones. Además, son necesarias investigaciones que aporten luz sobre este tipo de tratamientos: asi, ¿cuánto tiempo debe durar un tratamiento?, ¿cuántas sesiones?, ¿qué resulta más recomendable, la realización de sesiones espaciadas o por el contrario, muy seguidas?, ¿existe algún perfil concreto de pacientes esquizofrénicos susceptibles de beneficiarse de este tipo de intervención?, ¿en qué momento de la evolución clínica deben aplicarse?, ¿cuál es la estabilidad temporal de los resultados alcanzados?, ¿se produce algún proceso de generalización, ¿de qué tipo?...son algunas de las preguntas que necesitan respuesta.

En trastornos mentales que precisan de tratamientos complejos, es conveniente poder contar con la colaboración de otros agentes (familiares, monitores de talleres ocupaciona- les, etc). Esto facilita la aplicación de intervenciones multimodales, asi como la valoración de sus resultados. Al mismo tiempo, implica la ne-cesidad de prepararles para el adecuado desempeño de las tareas que se les encomiende.

La evaluación conductual de los sintomas psicóticos esquizofrénicos es una alternativa válida y compatible con el diagnóstico psiquiátrico. Se trata de un enfoque que se aleja de la mera descripción de síntomas que posteriormente son homogeneizados en una etiqueta diagnóstica, pues ello conduce a la consideración de que toda la variedad y complejidad de tales conductas responde a una misma e hipotética causa. Por otro lado, se cuestiona que esas causas sean necesaria o exclusivamente factores internos (biologicos o mentales) desligados de la acción o influencia del entorno físico, social y cultural donde vive el sujeto (A)Issa, 1977).

El modelo conductual propone el análisis funcional de los sintomas psicóticos positivos y negativos, síntomas que con frecuencia están funcionalmente interrelacionados entre sí, además de con otras variables de carácter situacional y organismico (Cuevas y Perona, 1995). Por tanto, ideas delirantes y alucinaciones son con-ductas, o sintomas, con entidad propia de cara a ser considerados como elementos objeto de análisis e intervención, independientemente de que sean elementos constitutivos de un síndrome psiquiátrico. Desde esta posición resulta posible el diseño de intervenciones contextualizadas y dirigidas a la modificación de la sintomatología clinica del sujeto y a la consecución de un mejor funcionamiento psicosocial en su entorno. Además como sugiere Persons (1986), los enfoques basados en el análisis del síntoma favorecen el conocimiento y el desarrollo teórico de los mismos, disminuyendo el riesgo de quedar olvidados en categorias sindromicas.

\section{REFERENCIAS BIBLIOGRÁFICAS}

Al-Issa, I. (1977). Social and cultural aspects of hallucinations. Psychological Bulletin, 84 (3), 570-587.

Alford, B.A. y Beck, A.T. (1994). Cognitive therapy of delusional beliefs. Behavioral Research and Therapy, 32, vol. 32, 3, 369-380. 
American Psychiatric Association. (1988). DSM-III$R$ : Manual diagnóstico y estadistico de los trastornos mentales. Barcelona: Masson, S.A. (orig. 1987).

American Psychiatric Association. (1995). DSM-IV: Manual diagnóstico y estadistico de los trastornos mentales. Barcelona: Masson, S.A. (orig. 1994).

Baños, R.M. y Belloch, A. (1995). Psicopatologia del pensamiento (II): los delirios. En A. Belloch, B. Sandín y F. Ramos: Manual de psicopatologia. Vol.1, 303-334. Madrid: McGraw-Hill/ Interamericana de España.

Beck, A.T. (1979). Cognitive therapy and emotional disorders. Nueva York: New American Library.

Beck, A.T. Rush, A.J. Shaw, B.F. y Emery, G. (1979). Cognitive theraphy of depression. New York: Guilford Press.

Bentall, R.P. (1990). The illusion of reality: a review and integration of psychological research on hallucinations. Psychological Bulletin, 107, No. 1, 82-95.

Birchwood, M. y Tarrier, N. (1995). El tratamiento psicológico de la esquizofrenia. Barcelona: Ariel psicología.

Brett-Jones, J. Garety, P. y Hemsley, D. (1987). Measuring delusional experiences: a method and its application. British Journal of Clinical Psychology, 26, 257-265.

Chadwick, P.D.J. y Lowe, C.F. (1994). A cognitive approach to measuring and modifying delusions. Behavioral Research and Therapy, 32, vol. 32, 3, 355-367.

Chadwick, P. Birchwood, M. y Trower, P. (1996). Cognitive therapy for delusions, voices and paranoie. Chichester: Wiley.

Cooklin, R. Sturgeon, D. y Leff, J. (1983). The relationship betwen auditory hallucinations and spontaneous fluctuations of skin conductance in schizophrenia. British Joumal of Psychiatry, 142, 47-52.

Cuevas, C. y Perona, S. (1990). Historia de Rehabilitación Psicosocial de la Unidad "Virgen del Rocio". Unidad de Rehabilitación "Virgen del Rocio", Sevilla (documento no publicado).

Cuevas, C. y Perona, S. (1995). Evaluación conductual de la esquizofrenia. Apuntes de Psicologia, 43, 45-63.
Fernández Ballesteros, R. y Carrobles, J.A.I. (1981). Evaluación conductual: metodología y aplicaciones. Madrid: Pirámide.

Fowler, D. (1996). Terapia cognitivo-conductual: un nuevo enfoque para el manejo de los trastomos psicóticos. En Jose Antonio Aldaz y Carmelo Vázquez (comps.) Esquizofrenia: fundementos psicológicos y psiquiátricos de la rehabilitación. Cap. $n^{\circ}$ 10, 187-204. Madrid: Siglo XXI.

Kingdon, D.G. y Turkington, D. (1995). CognitiveBehavioral therapy of schizophrenia. Psychology Press (orig. 1994).

Lowe, C.F. y Chadwick, P.D.J. (1990). Verbal control of delusions. Behavior Therapy, 21, 461479.

Lukoff, D. Nuechterlein, K.H. y Ventura, J. (1986). Manual for expanded. Brief Psychiatric Rating Scale (BPRS). Schizophrenia Bulletin, 12, 594602.

Maher, B.A. (1988). Anoumalous experience and delusional thinking: the logic explanation. En T.F. Oltmanns y B.A. Maher (eds.). Delusional beliefs, 15-33. New York: Wiley.

Perona, S. y Cuevas, C. (1997). Tratamiento conductual de las alucinaciones auditivas en un paciente esquizofrénico: estudio de caso. Psicothema, vol. 9, 1, 33-45.

Persons, J. (1986). The advantages of studying psychological phenomena rather than psychiatric diagnoses. American Psychologist, 41, 1252. 1260.

Segura, M. Sánchez, P. y Barbado, P. (1991). Análisis funcional de la conducta: un modelo explicativo. Granada: Universidad de Granada.

Siegel, J.M. (1975). Successful systematic desensitization in a chronic schizophrenic patient. Journal Behavioral Therapy \& Experimental Psychiatry, 6, 345-346.

Wallace, C.H. Boone, S. Donahoe, C. y Foy, D. (1985). The chronically mentally disabled: independent living skills training. En D.G. Barlow: Clinical Handbook of Psychological Disorders. New York: Guilford Press. 